\title{
Environmental issues affected women's caring
}

Wuest J. Illuminating environmental influences on women's caring.J Adv Nurs 1997 Jul;26:49-58.

\section{Objective}

To provide an exploratory theory of women's caring within the larger sociopolitical environment.

\section{Design}

Feminist grounded theory.

\section{Setting}

Rural and urban areas of a province in eastern Canada.

\section{Patients}

21 women who were caring for well, physically or mentally handicapped, acute and chronically ill selves, partners, children, or other family members were studied. Women from diverse socioeconomic situations, cultures, sexual orientations, educational backgrounds, and abilities were included.

\section{Methods}

Data were collected using individual or group interviews, participant observation at self help groups and health information sessions, and previous interviews with parents.

\section{Main results}

The most difficult feature of caring was the competing and changing demands from themselves and others. Women managed the demands using a dynamic process named precarious ordering that involved moving from relative disorder (reactive stage) of fraying connections to relative order (proactive stage) of setting boundaries, negotiating, and repatterning care (fig).

An important discovery was the complex reciprocal influence of environmental conditions and the process of precarious ordering. The salient internal and external environmental conditions were caring ideals, caring proximity, caring options, and caring rewards. Caring ideals were images of caring held by the women, family members, the community, and the system. Proximity was the geographic, relational, and cultural closeness or distance between women and care recipients and those who assisted in that caring. Options for caring hinged on availability and suitability of financial, psychosocial, and material resources. Caring rewards were the internal satisfactions, external feedback, and concrete achievements that sustained women's caring.

\section{Conclusions}

The theory of precarious ordering expands nursing's understanding of the interplay between the sociopolitical structure and caring by identifying environmental variables that influence caring.

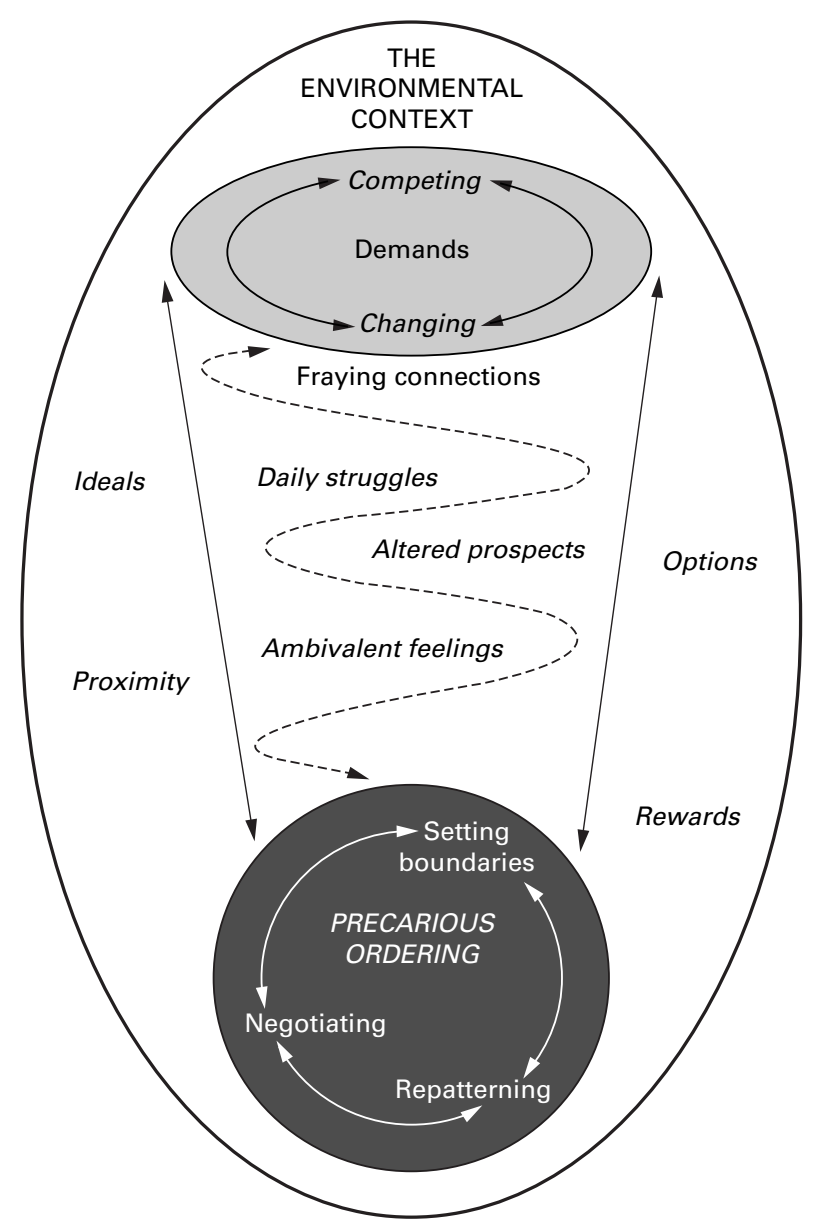

A depiction of the process of precarious ordering reproduced with permission from Journal of Advanced Nursing 1997;26:49-58.

Source of funding: The Dorothy J Kergin Research Grant in Primary Health Care, Canadian Nurses Foundation.

For article reprint:Dr J Wuest, Faculty of Nursing, University of New Brunswick, Fredericton, New Brunswick E3B 5A3, Canada. Fax +15064473057.

\section{Commentary}

As healthcare systems cut back services in efforts to reduce costs, increasing pressure may be placed on women to take on additional caring responsibilities. Research related to caring has examined the positive outcomes of caring, ${ }^{1}$ and the problems including caregiver burden and strain, particularly with people who are elderly and demented. ${ }^{2}$ Little research, however, has examined women's caring within the existing physical, cultural, political, and social environment.

Feminist grounded theory is a methodology that permits sharing of personal experiences. The theory developed by Wuest is easily understood and the components of the theory are aptly named. The dynamic process of precarious ordering, fraying connections and the proactive stage of setting boundaries, negotiating, and repatterning are understood from experience alone.

The opinions and needs expressed by the women expand understanding of the complexity of the caring experience. Nurses must represent a true partnership in recognising the environmental influences women have to negotiate and their ability to do so. The need for information on available resources could be facilitated by nurses.

Nurses must have information on cross cultural nursing to assist them in understanding the expectations of other cultures about caring behaviours. Nurses can also support women who care for families with different cultural beliefs.

Nurses could work collaboratively to lobby governments for the recognition of the need for additional services for women. Nurses can articulate the caring behaviours provided by women and their resource needs. As the caring demands on women increase, the availability of appropriate resources within the social structure will be of vital importance.

Gloria Harrison, RN, MHSc Assistant Executive Director, Nursing Norfolk General Hospital Simcoe, Ontario, Canada

1 Roach S. The human act of caring. Ottawa: Canadian Hospital Association, 1992. 2 Given B, Given C. Ann Rev Nurs Res 1991;9:77-
99. 